\title{
Histologic changes mimicking biliary disease in liver biopsies with venous outflow impairment
}

\author{
Sanjay Kakar ${ }^{1}$, Kenneth P Batts², John J Poterucha ${ }^{3}$ and Lawrence J Burgart ${ }^{4}$ \\ ${ }^{1}$ Department of Pathology, Veteran Affairs and University of California Medical Center, San Francisco, CA, \\ USA; ${ }^{2}$ Department of Pathology, Abbott Northwestern Hospital, Minneapolis, MN, USA; ${ }^{3}$ Department of \\ Gastroenterology and ${ }^{4}$ Department of pathology, Mayo Clinic, Rochester, MN, USA
}

\begin{abstract}
Impairment of venous outflow from the liver manifests as zone 3 sinusoidal dilatation and congestion (SDC) in liver biopsy. The spectrum of histologic changes in portal tracts has not been described. We studied liver biopsies from 34 patients with a confirmed diagnosis of venous outflow impairment (VOI). Liver transplant recipients and biopsies with cirrhosis and hepatic neoplasms were excluded. Clinical records were reviewed for laboratory tests and radiographic findings. In all, 19 patients had right heart disease, 13 had classic BuddChiari syndrome and two had veno-occlusive disease. Liver chemistry tests showed elevated liver transaminases $(n=21 ; 61.8 \%)$, elevated alkaline phosphatase $(n=31 ; 91.2 \%)$ and GGT (all 13 cases tested). The elevation in ALT and AST was mild (below $200 \mathrm{U} / \mathrm{l}$ in all cases), while alkaline phosphatase (ALP) was elevated above $500 \mathrm{U} / \mathrm{l}$ in nine (26.5\%) patients and above $1000 \mathrm{U} / \mathrm{l}$ in three cases. On biopsy, all cases showed SDC. The portal tracts showed (a) portal expansion with bile ductular proliferation $(n=16 ; 47.1 \%)$ accompanied by lymphoplasmacytic infiltrate $(n=10)$, lymphocytic cholangitis $(n=3)$ and portal or periportal fibrosis $(n=11)$, (b) Portal and/or periportal fibrosis without ductular proliferation $(n=3 ; 8.8 \%)$ or (c) Normal portal tracts $(n=15$; 44.1\%). The combination of elevated ALP and bile ductular changes on biopsy suggested chronic bile duct disease. Ultrasound/CT scan evaluation of bile ducts in 26 patients showed no biliary tree abnormality. Antimitochondrial antibody testing in eight cases also yielded negative results. In conclusion, bile ductular proliferation, portal inflammation and portal-based fibrosis are commonly seen in liver biopsies of patients with VOI even in the absence of bile duct disease. These changes are often accompanied by elevated ALP and GGT and can lead to the suspicion of chronic biliary disease. In the absence of demonstrable abnormalities in the biliary tree, these changes can be attributed to venous outflow impairment.
\end{abstract}

Modern Pathology (2004) 17, 874-878, advance online publication, 16 April 2004; doi:10.1038/modpathol.3800073

Keywords: sinusoidal dilatation; Budd-Chiari; portal fibrosis; bile ductular proliferation

Impairment of venous outflow from the liver can result from obstruction of the hepatic veins or inferior vena cava and due to cardiac causes like right-sided heart disease and constrictive pericarditis. ${ }^{1-5}$ On liver biopsy, histologic changes include zone 3 sinusoidal dilatation and congestion, red blood cell extravasation in the space of Disse and hepatocyte atrophy. With chronic outflow impairment, fibrosis can develop starting in zone 3 . These changes are well described in several articles and details of the changes in sinusoidal blood flow and mechanism of central-central and central-portal

Correspondence: LJ Burgart, MD, 200 First Street SW, Department of Pathology, Rochester, MN 55905, USA.

E-mail: lburgart@mayo.edu

Received 25 July 2003; revised 3 October 2003; accepted 5 October 2003; published online 16 April 2004 fibrosis have been studied. ${ }^{2,3,6-11}$ However, histologic features in portal tracts in liver biopsies with venous outflow impairment have not been detailed in any of these publications ${ }^{1-11}$ or standard liver textbooks. ${ }^{12,13}$ In our experience, abnormal portal tracts are often seen in venous outflow impairment and can mimic the findings of chronic biliary disease. These patients often have elevated alkaline phosphatase (ALP) and gamma glutamyl transferase (GGT) with mild elevation of liver aminotransferases. Thus liver enzyme tests as well as the histopathologic findings can raise the suspicion of chronic biliary disease and may lead to unnecessary diagnostic work-up. The purpose of this study is to characterize the spectrum and significance of pathologic changes in portal tracts in liver biopsies with hepatic venous outflow impairment. 


\section{Methods}

All available liver biopsies with a confirmed diagnosis of venous outflow impairment obtained at our institution between 1994 and 2001 were retrieved. Liver transplant recipients and biopsies with cirrhosis were excluded. The biopsies were reviewed to confirm the diagnosis, and the following features were recorded:

1. Adequacy (number of portal tracts).

2. Bile ductular proliferation (absent/present/ marked).

3. Bile duct damage (absent/present).

4. Portal inflammation: intensity (mild, moderate, marked) and cell types. (lymphocytes, plasma cells, neutrophils, eosinophils).

5. Fibrosis(sinusoidal, portal, periportal, septal).

6. Degree of sinusoidal dilatation and congestion: mild (zone 3), moderate (zones 3 and 2), marked (zones 1, 2 and 3).

The clinical records of the patients were reviewed for the following characteristics:

1. Age and gender.

2. Duration of symptoms: acute $(<1$ month), chronic ( $>1$ month).

3. Liver chemistry tests: bilirubin, liver enzymes (ALT, AST, alkaline phosphatase, GGT), prothrombin time, serum albumin.

4. Viral serologies for hepatitis A, B and C.

5. Serum autoantibodies: antinuclear (ANA), antismooth muscle (SMA), antimitochondrial (AMA).

6. Radiological tests: ultrasound/CT scan/MRI of the liver, endoscopic retrograde cholangiopancreatography (ERCP) with cholangiogram.

Fisher's exact test and $\chi^{2}$ tests were used for statistical analysis.

\section{Results}

The search results yielded 141 liver biopsies with sinusoidal dilatation and congestion. Of these, 107 cases were excluded: 90 were biopsies from liver transplant recipients and cirrhotic patients, and 17 were cases with SDC without clinical and/or radiologic evidence of venous outflow impairment. The remaining 34 patients had confirmed clinical and/or radiological evidence of venous outflow impairment and formed the study group. Of these, 19 had right heart disease, 13 had classic BuddChiari syndrome due to obstruction of hepatic veins or inferior vena cava, and two had veno-occlusive disease (VOD). The 19 patients with heart disease included tricuspid valve disease $(n=1)$, tricuspid and mitral valve disease $(n=3)$, complex cyanotic heart disease $(n=1)$, idiopathic dilated cardiomyopathy $(n=5)$, cardiac amyloidosis $(n=7)$, chronic ischemic heart disease $(n=1)$ and constrictive pericarditis $(n=1)$. The various associations observed with Budd-Chiari syndrome were polycythe- mia vera $(n=2)$, oral contraceptives $(n=2)$ and one case each of paroxysmal nocturnal hemoglobinuria and chronic myeloproliferative disorder. In all, seven cases were idiopathic. The diagnosis of Budd-Chiari syndrome was made on the basis of clinical presentation (features of hepatic congestion like hepatomegaly and ascites, absence of other demonstrable causes of acute or chronic liver disease, presence of predisposing hypercoaguable states) along with radiologically demonstrated occlusion of hepatic veins $(n=9)$ and/or liver biopsy showing SDC $(n=13)$. Of the two cases with VOD, one patient had been treated for acute lymphoblastic leukemia (ALL) and the second had received a bone marrow transplant for multiple myeloma.

\section{Clinical and Laboratory Findings (Table 1)}

The presentation was acute (symptom duration 1 month or less at presentation) in seven (20.6\%) patients with symptom duration of 1 month or less at presentation, and chronic in $22(64.7 \%)$ patients. The type of presentation was not known in five patients. Laboratory findings included elevated

Table 1 Clinical and laboratory features seen with different portal tract changes in venous outflow impairment

$\begin{array}{ccc}\text { Portal } & \text { Portal } & \text { Normal P-value } \\ \text { tracts } & \text { tracts } & \text { portal } \\ \text { with } & \text { with } P F^{\mathrm{b}} & \text { tracts } \\ B D P^{\mathrm{a}} & \text { without } & \mathrm{n}=15 \\ \mathrm{n}=16 & B D P^{\mathrm{c}} & \\ & \mathrm{n}=3 & \end{array}$

\begin{tabular}{|c|c|c|c|c|}
\hline \multicolumn{5}{|l|}{ Etiology } \\
\hline Cardiac & 9 & 1 & 9 & \multirow[t]{3}{*}{0.5} \\
\hline BCS & 5 & 2 & 6 & \\
\hline VOD & 2 & 0 & 0 & \\
\hline \multicolumn{5}{|c|}{ Symptom duration $(\mathrm{n}=29)^{\mathrm{c}}$} \\
\hline$<1$ month & 3 & 1 & 3 & \multirow[t]{2}{*}{0.9} \\
\hline$>1$ month & 9 & 2 & 11 & \\
\hline \multicolumn{5}{|l|}{ Bilirubin } \\
\hline Normal & 1 & 1 & 2 & \multirow[t]{2}{*}{0.3} \\
\hline Elevated & 15 & 2 & 13 & \\
\hline \multicolumn{5}{|l|}{$A L T / A S T$} \\
\hline Normal & 6 & 1 & 6 & \multirow[t]{2}{*}{0.9} \\
\hline Elevated & 10 & 2 & 9 & \\
\hline \multicolumn{5}{|l|}{$G G T(\mathrm{n}=13)$} \\
\hline Normal & 0 & 0 & 0 & \multirow[t]{2}{*}{ na } \\
\hline Elevated & 11 & 1 & 1 & \\
\hline \multicolumn{5}{|l|}{$A L P$} \\
\hline Normal & 1 & 0 & 2 & \multirow[t]{2}{*}{0.6} \\
\hline Elevated & 15 & 3 & 13 & \\
\hline \multicolumn{5}{|c|}{ Viral serologies $(\mathrm{n}=22)$} \\
\hline Negative & 14 & 1 & 7 & \multirow[t]{2}{*}{ na } \\
\hline Positive & 0 & 0 & 0 & \\
\hline \multicolumn{5}{|l|}{$A M A(\mathrm{n}=8)$} \\
\hline Negative & 6 & 0 & 2 & \multirow[t]{2}{*}{ na } \\
\hline Positive & 0 & 0 & 0 & \\
\hline
\end{tabular}

BCS, Budd-Chiari syndrome; VOD, veno-occlusive disease; ALT, alanine aminotransferase; AST, aspartate aminotransferase; ALP, alkaline phosphatase; GGT, gamma glutamyl transferase; AMA, antimitochondrial antibodies; na, not applicable.

${ }^{a}$ Bile ductular proliferation; ${ }^{b}$ portal-based fibrosis; 'duration unknown in five patients. 
bilirubin $(n=30 ; 88.2 \%)$, elevated liver transaminases $(n=21 ; 61.8 \%)$ and elevated alkaline phosphatase $(n=31 ; 91.2 \%)$. GGT was high in all 13 cases tested. The elevation in ALT and AST was mild (below 200 U/l in all cases), while ALP was elevated above $500 \mathrm{U} / \mathrm{l}$ in nine $(26.5 \%)$ patients and above $1000 \mathrm{U} / \mathrm{l}$ in three $(8.8 \%)$ cases. Antimitochondrial antibodies were negative in all in eight cases tested.

\section{Liver Biopsy (Table 2)}

All the biopsies were adequate, with four or more portal tracts. All showed zone 3 sinusoidal dilatation and congestion with hepatic plate atrophy. The morphologic changes in the portal tracts fell into three categories:

(a) portal expansion with bile ductular proliferation $(n=16 ; 47.1 \%)$. The proliferation was

Table 2 Histologic findings in portal tracts in liver biopsies in patients with venous outflow impairment

\begin{tabular}{lcccc}
\hline Portal tract histology & $\begin{array}{c}\text { Cardiac } \\
\text { causes } \\
(\mathrm{n}=19)\end{array}$ & $\begin{array}{c}\text { Budd- } \\
\text { Chiari } \\
\text { syndrome } \\
(\mathrm{n}=13)\end{array}$ & $\begin{array}{c}\text { Veno- } \\
\text { occlusive } \\
\text { disease } \\
(\mathrm{n}=2)\end{array}$ & P-value \\
\hline $\begin{array}{l}\text { Bile ductular proliferation } \\
(n=16)\end{array}$ & 8 & 6 & 2 & 0.3 \\
$\begin{array}{l}\text { Lymphocytic cholangitis } \\
(n=2)\end{array}$ & 1 & 1 & 0 & 0.6 \\
$\begin{array}{l}\text { Portal inflammation } \\
(n=13)\end{array}$ & 7 & 5 & 1 & 0.9 \\
$\begin{array}{l}\text { Portal-based fibrosis } \\
(n=14)\end{array}$ & 8 & 4 & 2 & 0.2 \\
$\begin{array}{l}\text { Sinusoidal fibrosis } \\
(n=21)\end{array}$ & 10 & 9 & 2 & 0.3 \\
\hline
\end{tabular}

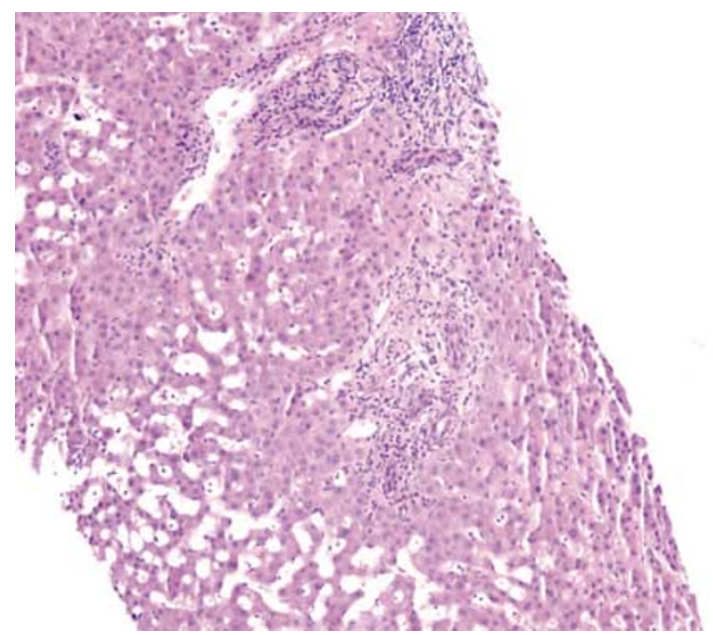

Figure 1 Sinusoidal dilatation and congestion in zones 2 and 3. Portal tracts show mild bile ductular proliferation and a mild lymphocytic infiltrate, hematoxylin and eosin, $\times 100$. mild in 14 cases (Figures 1 and 2) and moderate to marked in two cases (Figure 3). Cholestasis or ductopenia were not observed in any case. This was accompanied by a mild lymphoplasmacytic infiltrate in 10 cases, of which two cases showed lymphocytic cholangitis (Figure 4). A few neutrophils were often seen in association with proliferating ductules. Portal-based fibrosis was seen in 11 biopsies: portal fibrosis in six cases, periportal fibrosis in three cases and septal fibrosis in two cases. Histologic changes reflecting cholate stasis like pseudoxanthomatous change and Mallory's hyaline were not observed in any case.

(b) Portal-based fibrosis without bile ductular proliferation $(n=3 ; 8.8 \%)$. All three cases showed periportal fibrosis with a mild lymphoplasmacytic inflammatory infiltrate (Figure 5).

(c) Normal portal tracts $(n=15 ; 44.1 \%)$.

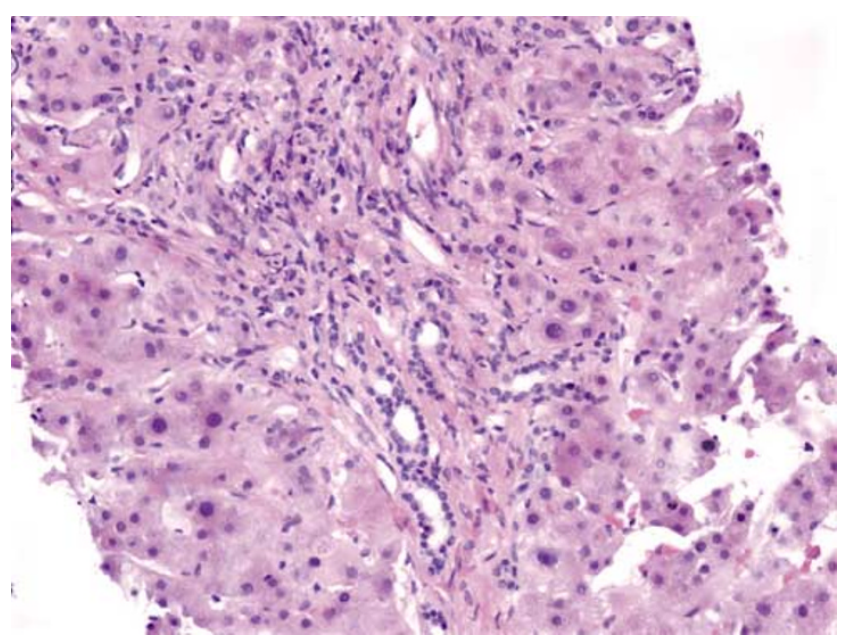

Figure 2 Portal expansion with mild bile ductular proliferation, hematoxylin and eosin, $\times 200$.

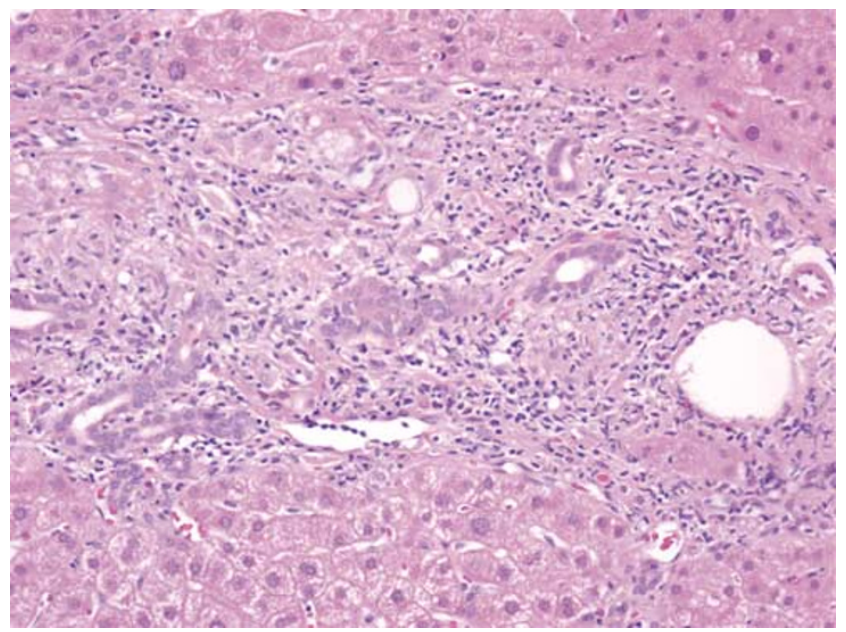

Figure 3 Marked bile ductular proliferation, hematoxylin and eosin, $\times 200$. 


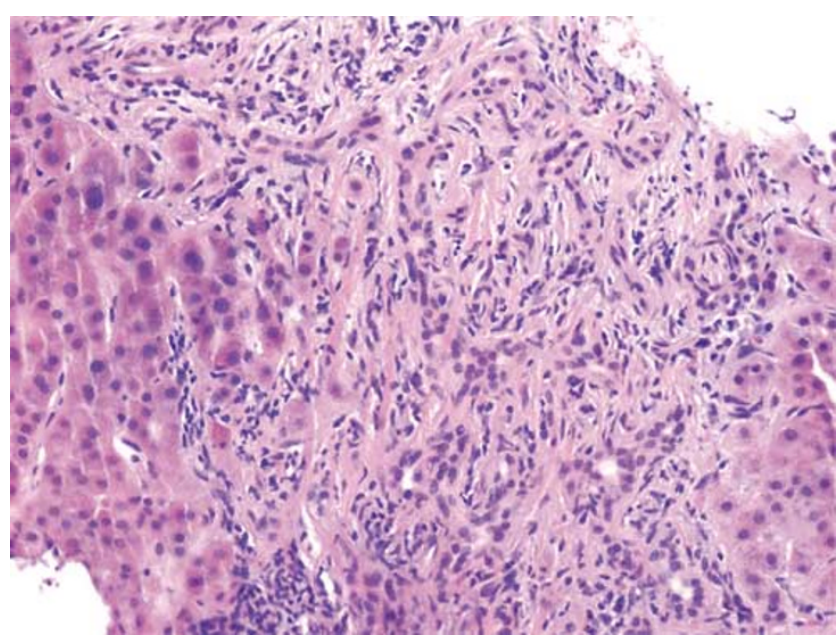

Figure 4 Bile ductular proliferation with lymphocytic cholangitis and periportal fibrosis, hematoxylin, and eosin, $\times 200$.

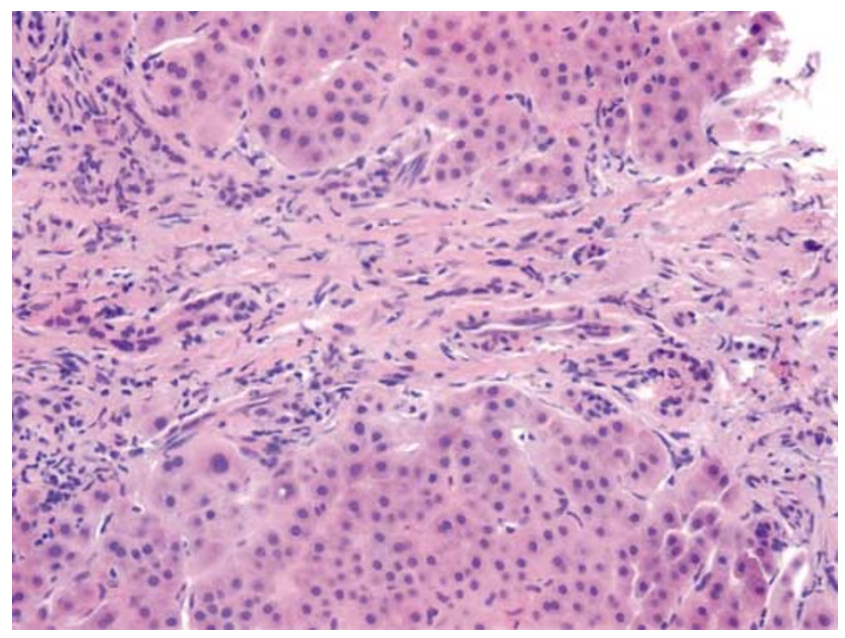

Figure 5 Periportal fibrosis without significant bile ductular proliferation, hematoxylin and eosin, $\times 200$.

There was no significant difference among the three morphologic groups in terms of etiology of venous outflow impairment $(P=0.5)$, duration of symptoms $(P=0.9)$, bilirubin levels $(P=0.3)$ and ALT/AST $(P=0.9)$ and alkaline phosphatase $(P=0.6)$.

Of the seven patients with acute presentation, three had portal-based fibrosis and five had sinusoidal fibrosis. Of 22 patients with chronic presentation, 11 had portal-based fibrosis and 14 had sinusoidal fibrosis. The duration of symptoms did not correlate with the presence of fibrosis $(P=0.5)$.

\section{Radiology}

Evaluation of bile ducts with ultrasound or CT scan was available in 26 patients, none of whom showed a biliary tree abnormality. ERCP was not performed in any case.

\section{Discussion}

The diagnosis of venous outflow impairment of the liver is based on clinical and radiographic findings, and liver biopsy can be performed to confirm the diagnosis or assess the degree of fibrosis. Many studies have described the histopathologic findings in hepatic venous outflow impairment. ${ }^{2,3,6-11}$ Most patients show evidence of chronic passive congestion, which manifests as sinusoidal dilatation and varying degree of hepatic atrophy and necrosis. The congestion and necrosis are most marked in zone 3 of the acinus, but can involve zones 1 and 2 in more severe cases. Extravasation of red blood cells in the space of Disse has also been described. ${ }^{9}$ In chronic cases, these changes are accompanied by zone 3 sinusoidal fibrosis, which can progress to bridging fibrosis and cirrhosis. In all these reports, the description of liver pathology emphasizes the changes in liver sinusoids and hepatic parenchyma, and the patterns of fibrosis that are seen with chronic congestion. However, the morphological changes in portal tracts in this setting are not mentioned in these reports. Intrahepatic biliary tree abnormalities in Budd-Chiari syndrome detected by endoscopic retrograde cholangiopancreatography have been described in a case study. ${ }^{14}$ These changes included crowding and elongation of the bile ducts and dilatation of some of the distal bile duct radicles. However, the bile duct changes in liver biopsy were not mentioned.

In our pathology consultation practice, cases with typical morphologic findings of venous outflow impairment are often sent for a second opinion due to the presence of portal tract changes that mimic a biliary disease. In the present study, nearly half of the cases showed bile ductular proliferation in portal tracts often accompanied by portal-based fibrosis and mild lymphoplasmacytic inflammation. Occasionally, the bile ductular proliferation was florid and evidence of bile duct damage in the form of lymphocytic cholangitis was seen. In a few instances, portal-based fibrosis and mild lymphoplasmacytic inflammation were seen without bile ductular proliferation. These morphologic changes can easily be interpreted as indicating chronic biliary disease.

The liver enzyme profile also resembles that of biliary disease. ALP was elevated in almost all the cases and was accompanied by elevated GGT (all 13 cases tested) and mildly elevated ALT/AST levels $(<200 \mathrm{U} / \mathrm{l}$ in all cases). The liver enzyme results along with the histopathologic findings of bile ductular proliferation, portal inflammation and portal/periportal fibrosis can raise a strong suspicion of a biliary etiology. In this series, the possibility of chronic biliary disease was raised in the original pathology report in five cases. AMA testing was negative (all six cases tested) and ultrasound/CT scan evaluation of bile ducts in 26 cases failed to reveal any abnormality in the biliary 
tree. There was no significant correlation between the presence of portal tract morphologic changes and etiology of venous outflow impairment, duration of symptoms, bilirubin and ALT/AST levels. ALP levels exceeding $1000 \mathrm{U} / \mathrm{l}$ were seen in three patients with abnormal portal tracts but none of the patients with normal portal tracts on biopsy. This difference was not statistically significant.

In our study, the proportion of cases with sinusoidal and portal-based fibrosis was nearly equally distributed among the patient groups with acute and chronic disease presentations. It is intuitive to assume that patients with chronic symptoms will have more fibrosis compared to those with an acute presentation. However, no correlation was identified between the duration of symptoms and presence of sinusoidal or portalbased fibrosis. Previous studies have reported a similar lack of correlation between the degree of sinusoidal fibrosis and duration of symptoms. ${ }^{2,3}$ It is thought that patients with Budd-Chiari syndrome who present in an acute manner have had relatively long-standing subclinical obstruction, accounting for the presence of fibrosis in many of these cases.

The mechanism of bile ductular proliferation and portal-based fibrosis in venous outflow impairment is not known. There may be a low-grade chronic injury to the biliary system that accounts for these changes. Sinusoidal dilatation and increased venous pressure or compromised arterial flow due to abnormal shunts may lead to a low level ischemic damage. Ischemic injury is implicated in rare cases of biliary abnormalities (portal biliopathy) that develop in extrahepatic portal hypertension. ${ }^{15}$ Bile ductular proliferation has been described in association with large regenerative nodules in Budd-Chiari syndrome. ${ }^{6}$ The benign regenerative nodules in Budd-Chiari syndrome can show copper accumulation indicating some sort of biliary perturbation. ${ }^{16}$

As shown by our results, these changes are often seen in the absence of regenerative nodules.

In summary, portal tract changes in the form of bile ductular proliferation, lymphoplasmacytic inflammation and portal/periportal fibrosis are commonly encountered in liver biopsies with venous outflow impairment. These changes closely resemble the features of chronic biliary disease, a suspicion that is heightened by the frequent elevation of alkaline phosphatase and gamma-glutamyl transferase in these patients. Serological and radiological tests for biliary disease were normal in all cases in our study. Awareness of these morphologic changes can prevent overemphasizing the risk of biliary disease in this setting and prevent unnecessary diagnostic evaluation.

\section{References}

1 Valla DC. Hepatic vein thrombosis (Budd-Chiari syndrome). Semin Liver Dis 2002;22:5-14.

2 Dilawari JB, Bambery P, Chawla Y, et al. Hepatic outflow obstruction (Budd-Chiari syndrome). Experience with 177 patients and a review of the literature. Medicine (Baltimore) 1994;73:21-36.

3 Bhusnurmath SR. Budd-Chiari syndrome: current concepts. Indian J Gastroenterol 1994;13:9-12.

4 Naschitz JE, Slobodin G, Lewis RJ, et al. Heart diseases affecting the liver and liver diseases affecting the heart. Am Heart J 2000;140:111-120.

5 Wanless IR, Liu JJ, Butany J. Role of thrombosis in the pathogenesis of congestive hepatic fibrosis (cardiac cirrhosis). Hepatology 1995;21:1232-1237.

6 Tanaka M, Wanless IR. Pathology of the liver in BuddChiari syndrome: portal vein thrombosis and the histogenesis of veno-centric cirrhosis, veno-portal cirrhosis, and large regenerative nodules. Hepatology 1998;27:488-496.

7 Iwai M, Kitagawa Y, Nakajima T, et al. Clinical features, image analysis, and laparoscopic and histological liver findings in Budd-Chiari syndrome. Hepatogastroenterology 1998;45:2359-2368.

8 Tang TJ, Batts KP, de Groen PC, et al. The prognostic value of histology in the assessment of patients with Budd-Chiari syndrome. J Hepatol 2001;35: 338-343.

9 Leopold JG, Parry TE, Storring FK. A change in the sinusoid-trabecular structure of the liver with hepatic venous outflow block. J Pathol 1970;100: 87-98.

10 Kage M, Arakawa M, Kojiro M, et al. Histopathology of membranous obstruction of the inferior vena cava in the Budd-Chiari syndrome. Gastroenterology 1992; 102:2081-2090.

11 Cho KJ, Geisinger KR, Shields JJ, et al. Collateral channels and histopathology in hepatic vein occlusion. Am J Roentgenol 1982;139:703-709.

12 Wanless IR. Vascular disorders. In: MacSween RNM, Burt AD, Portman BC, Ishak KG, Scheuer PJ, Anthony PP (eds). Pathology of the Liver, 4th edn. New York: Churchill Livingstone, Inc., 2002, pp 539-574.

13 Valla D, Benhamou JP. Obstruction of the hepatic venous system. In: Bircher J, Benhamou JP, McIntyre N, Rizzetto M, Rodes J (eds). Oxford Textbook of Clinical Hepatology, 2nd edn. New York: Oxford University Press, Inc., 1999, pp 1469-1478.

14 Moriera VF, del Olmo ML, Seara JF. La via biliar intrahepatica en el sindrome de Budd-Chiari. Rev Clin Esp 1986;179:162-163.

15 Chandra R, Kapoor D, Tharakan A, et al. Portal biliopathy. J Gastroenterol Hepatol 2001;16: 1086-1092.

16 Soler R, Rodriguez E, Pombo F, et al. Benign regenerative nodules with copper accumulation in a case of chronic Budd-Chiari syndrome: CT and MR findings. Abdom Imaging 2000;25:486-489. 